removed. The use of sulphapyridine in such cases has already been described (Earle, 1940b).

(c) Catfish.-The spines of the catfish of Trinidad may produce localized cellulitis, ulceration, or gangrene. Sulphanilamide should be given prophylactically if possible.

Case 11.-A negro aged 14, seen on January 7, 1939, when he showed a cellulitis of the right hand due to a prick from a catfish spine. He was given sulphapyridine, 3 grammes daily, for two days. Cure was reported on January 9.

(d) Barracouda.-Bites from this fish often suppurate, and should receive prompt sulphanilamide therapy.

Case 12.-A Venezuelan aged 40, seen on January 8, 1939, when he displayed an infected barracouda bite of three days' duration. The area bitten was surrounded by a zone of ulceration and cellulitis. Sulphanilamide, 1 gramme thrice daily, was given, and the wound was dressed with magnesium sulphate and glycerin. On January 11 marked improvement was noted, and cure was complete on January 18.

\section{Venomous Arthropoda}

(a) Insecta.-(i) Chigoe Distase.-Ulceration and cellulitis surrounding the gravid felsale chigoe (Tunga penetrans) may respond to sulphanilamide therapy. I have seen three such cases react satisfactorily in this manner. (ii) Culicoides Bites.-Prophylactic treatment of bites of various Culicoides-C. amazonius, $C$. furens, $C$. stellifer, and $C$. diabolicus (Myers, 1935 ; Adamson, 1939)-which in Trinidad may be the starting-point of a tropical ulcer, has already been described (Earle, 1940a).

(b) Arachnida.-Several cases of encrusted scabies (gale norvégienne) have responded well to sulphanilamide therapy combined with local treatment with mitigal (dimethyl-diphenylene disulphide).

Case 13.-An East Indian labourer aged 20, seen on March 10, 1940, when he had encrusted and septic scabies of the palms, abdomen, penis, and scrotum. Sulphapyridine, 3 grammes daily, together with mitigal locally, was given. Cured in ten days.

(c) Myriapoda.-Severe cellulitis and ulceration which may follow bites of the centipede (? Scolopendra gigantea) have been controlled by sulphanilamide. Four successful cases have been noted, of which one is here described.

Case 14.-A negro labourer aged 30 , seen on January 17 , 1940, with severe cellulitis of the left hand following a centipede bite on the previous day. He was given sulphanilamide, 1 gramme thrice daily. The infection subsided in four days.

\section{Beriberi}

Bearing in mind the possible association of a bacterial exotoxin with vitamin $B_{1}$ deficiency in the production of beriberi (Low, 1936; Joyeux and Sicé, 1937), I tried the effect of sulphanilamide in a case of $B_{1}$ avitaminosis associated with cardiac lesions and oedema of the feet.

Case 15.-A female East Indian aged 25, first seen on January 4, 1939, when she complained of tingling and loss of sensation in the legs and feet, swelling of the feet, dyspnoea on slight exertion, and general weakness. The date of onset of the disease was uncertain. Examination showed a poorly built woman. The lips and conjunctivae were pale, teeth carious, and tongue furred. Firm oedema of both feet, dilatation of the right heart, and systolic murmurs were present. Pulse rate 96, tension low. Sensation of light touch was impaired in both feet and calves. Ankle-jerks were sluggish, knee-jerks were present. Ankylostome ova were observed in the stools. No malarial parasites were found in the blood. Anti-hookworm treatment was started at once. Vitamin $B_{1}$. 5,000 units daily. was injected intramuscularly for a week, and sulphanilamide was given orally for five days. On January 7 the patient had less dyspnoea and less oedema of the feet. A second course of sulphanilamide was given after three days' interval and vitamin $\mathbf{B}_{1}$ every second day. The patient made an uninterrupted recovery.
It is of course useless to draw any conclusions from an isolated case history. However, in cases of infantile beriberi seen recently in the island of Nauru-the patients sometimes being desperately ill, showing head retraction, spasticity of the limbs, Kernig's sign, and mental confusion, in addition to liver enlargement and hydroceledramatic results often followed lumbar puncture and intrathecal and intramuscular injection of large doses of vitamin $B_{1}$ combined with sulphapyridine by mouth. The vitamin used alone appeared to produce far less satisfactory results. Confirmatory work is obviously necessary in this obscure condition.

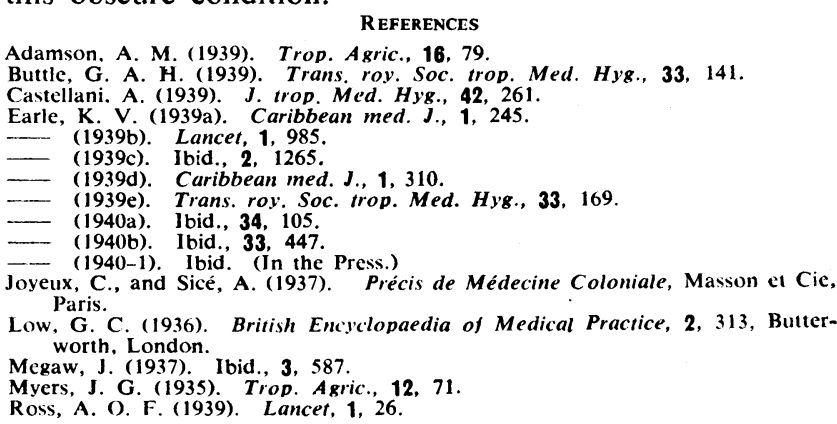

\section{LABORATORY INFECTION WITH BRUCELLA ABORTUS}

\author{
BY \\ H. N. GREEN, M.D., M.Sc. \\ Professor of Pathology, University of Sheffield
}

The report by Huddleson and Munger (1940) of an epidemic of brucellosis in Michigan State College due to laboratory infection with Brucella melitensis raises in a dramatic way the problem of dissemination and the possible portals of infection in man of the pathogenic Brucella group.

\section{Three Recent Cases}

In this connexion the experience in my laboratory is perhaps worth recording, although in this case infection was due to the less virulent $B r$. abortus. Case 1 (H. N. G.) arose two years after experimental work had been in progress with a strain of $\mathrm{Br}$. abortus originally isolated from a human case of undulant fever. The organism, after primary isolation in liver broth in an atmosphere of $10 \% \mathrm{CO}_{2}$, had been subcultured on plain media (agar or glycerin-agar) almost daily throughout this period, without any animal passage, and latterly grew rather better in air than in $10 \% \mathrm{CO}_{2}$. Careful precautions against accidental infection were taken, and the only direct exposure was in making frequent colonial counts from open agar plates, and in preparing and diluting small volumes of bacterial emulsions in saline from agar slopes. The attack of undulant fever did not arise after any unusual procedure or undue exposure, or after any known accidental contamination of the skin or laboratory benches. It followed a severe course, and though a trial of most of the therapeutic measures described was made, it subsided naturally after three months, immediately following the most severe pyrexial period of the illness. Sulphanilamide treatment was not, however, adequately carried out owing to sensitivity to the drug, which on one occasion produced an almost complete amblyopia lasting several hours. In the fourth week of convalescence a severe and protracted "myositis" of the lumbar muscles arose. There was no pyrexia, and the lesion was possibly allergic in nature.

Work was restarted with the same strain of organism two months after recovery, with more stringent precau- 
tions than before, particularly with regard to sterilization of the benches after contact with glassware containing $B r$. abortus cultures, hand and arm ablutions, and general alertness as to the risk of infection. Gloves were worn, possible contamination by splashing was rigorously guarded against, and the bench was covered with izal for twenty-four hours after each session. Nevertheless a further case arose in the senior laboratory assistant, eleven months after the onset of the first case (i.e., after subculture of the strain for three years). This person was exposed to the organism only in the periodic preparation, from Roux bottles, of culture washings, which were poured slowly through a very wide glass funnel into an Erlenmeyer flask. At the time of infection a sulphanilamide-resistant strain which had developed from the stock strain after culture in sulphanilamide broth (Green, 1940) was being grown in bulk in Roux bottles. It is of interest that the strain of $\mathrm{Br}$. abortus obtained from a blood culture previous to sulphapyridine treatment still retained about the same degree of resistance to sulphanilamide as the original infecting strain. This case ran a less severe course than the first, but did not become apyrexial for three months, though there was no serious relapse after giving an intensive course of sulphapyridine one month after the development of clinical symptoms.

The third case was that of another laboratory assistant, whose illness started one month after that of Case 2 . He had assisted Case 2 in preparing the washings from the Roux bottles, but had not been near a culture of $\mathrm{Br}$. abortus for one month, as all work with this organism was completely suspended and the preparation room was cleaned and disinfected when Case 2 was diagnosed. $\mathrm{He}$ also had a typical, fairly severe attack of undulant fever, which subsided naturally after about four months' illness.

\section{Possible Source of Infection}

There was, of course, nothing in the nature of an epidemic infection, and infection in every case may have occurred through some recognized channel such as a skin abrasion, droplet infection, or from contaminated fingers to the mouth. In all three cases, however, the individuals were not aware of any such contamination, and the second and third patients (in whom a great degree of caution had been induced by the occurrence and history of the first case) were certain that such contamination did not occur. Undoubtedly close contact with cultures was necessary for infection, as no case occurred in other members of the laboratory staff who were not working with the organism, whilst one member had assisted in the work for over two years without being clinically infected, though his serum agglutinated at one time at a titre of 1 in 100 .

It is suggested as a possibility (without any concrete evidence) that infection was due to inhalation of contaminated air in the vicinity of large concentrations of $\mathrm{Br}$. abortus growing on solid media. It is conceivable that, where the cultures had become rather dry, air currents (induced by Bunsen burners, etc.) could dislodge organisms from surface cultures. It should be noted also that Cases 2 and 3 were infected by a sulphanilamideresistant strain. A large proportion of the cultures to which Case 1 was exposed were also grown in and subcultured from media containing sulphanilamide. It is therefore possible that after exposure to sulphanilamide the organism had become more virulent or invasive than the stock strain.

\section{Commentary}

This record does not supplement our knowledge of the alternative paths of infection in man by the pathogenic Brucella group, which in the natural infection is commonly via the alimentary tract. It is published rather as a warning that $\mathrm{Br}$. abortus under laboratory conditions is a potentially highly dangerous organism and that the conditions governing its infectivity are still not completely understood. It is of particular interest that in Case 3 clinical symptoms did not appear until one month after any possible direct exposure to $\mathrm{Br}$. abortus cultures, which would fix the incubation period in this case at a minimum of one month.

The cause of the outbreak of Br. melitensis infection occurring at Michigan appears superficially much more mysterious than that described here, nor do the present observations throw any light on it, except to add some support to the possibility of air-borne infection. They do, however, indicate that there may be another quite unrecognized mode of infection, the nature of which, from both the academic and the practical standpoints, it would be of value to know. Progress might be made if the details of laboratory infection of all types were more often reported.

\section{Summary}

Three cases of undulant fever are reported, due to laboratory infection by a stock strain of Br. abortus which had been continuously subcultured for two to three years.

In two cases the infecting strain was known to be sulphanilamide-resistant.

In no case was the exact site of entry of the organism established, and it is suggested that air-borne infection from surface cultures is a possibility.

A grateful acknowledgment is due to Prof. E. J. Wayne, who undertook the medical care of two of the cases.

$$
\text { REFERENCES }
$$

Green, H. N. (1940). Brit. J. exp. Path., 21, 38 Huddleson, I. F., and Munger. M. (1940). Amer. J. publ. Hlth., 30, 944 (sec
Annotation. British Medical Journal, 1941, 1, 126).

\section{A CONVULSION DURING NON- CONVULSIVE FARADIC SHOCK THERAPY}

BY

GERALD CAPLAN, B.Sc., M.B., Ch.B.

House-physician at the City of Birmingham Mental Hospitai, Winson Green

Following on the promising results obtained by Berkwitz (1940) of Minneapolis, an investigation is being carried out on the therapeutic effect of non-convulsive faradic shock treatment in mental disease. This method is reputed to give as good results as the ordinary cardiazol or electrical convulsive therapy without the dangers consequent on the convulsions-namely, fractures of vertebrae, lesions of the brain, and abscesses of the lungreports of which are becoming ever more frequent (Polatin and others, 1939; Bennett and Fitzpatrick, 1939; Hamsa and Bennett, 1939 ; Blair, 1940).

The apparatus and technique used are very similar to those employed by Berkwitz. The faradic current is produced by an ordinary induction coil such as is used in most massage departments for muscle stimulation; and this is driven by a 6-volt dry battery. The electrodes are applied to the patient's temples, the current is switched full on, and then fifteen shocks of half a second's duration are given at intervals of half a second, varying the on-off key of the coil to make and break the current. During this period the patient is fully conscious. Each shock produces a spasmodic contraction of the muscles of the face and neck, and is described as feeling "like a bang on the head." 'Immediately after the series of shocks a $5 \%$ solution of pentothal sodium is injected intravenously. Enough is injected to produce a surgical anaesthesia lasting 5 to 10 minutes, and while the patient is coming round he is in an ideal state for psychotherapy. Apparently the faradic shock shakes him up and establishes 